\title{
sciendo
}

\section{Risk-Based Decision Support Model for Offshore Installations}

\author{
Gencer Erdogan, Atle Refsdal \\ SINTEF Digital, Oslo, Norway \\ Bjørn Nygård, Ole Petter Rosland \\ Statoil ASA, Stavanger, Norway \\ Bernt Kvam Randeberg \\ Oilfield Technology Group, Sandnes, Norway
}

\section{Abstract}

Background: During major maintenance projects on offshore installations, flotels are often used to accommodate the personnel. A gangway connects the flotel to the installation. If the offshore conditions are unfavorable, the responsible operatives need to decide whether to lift (disconnect) the gangway from the installation. If this is not done, there is a risk that an uncontrolled autolift (disconnection) occurs, causing harm to personnel and equipment. Objectives: We present a decision support model, developed using the DEXi tool for multi-criteria decision making, which produces advice on whether to disconnect/connect the gangway from/to the installation. Moreover, we report on our development method and experiences from the process, including the efforts invested. An evaluation of the resulting model is also offered, primarily based on feedback from a small group of offshore operatives and domain experts representing the end user target group. Methods/Approach: The decision support model was developed systematically in four steps: establish context, develop the model, tune the model, and collect feedback on the model. Results: The results indicate that the decision support model provides advice that corresponds with expert expectations, captures all aspects that are important for the assessment, is comprehensible to domain experts, and that the expected benefit justifies the effort for developing the model. Conclusions: We find the results promising, and believe that the approach can be fruitful in a wider range of risk-based decision support scenarios. Moreover, this paper can help other decision support developers decide whether a similar approach can suit them.

Keywords: safety risk, operational risk, offshore, multi-criteria decision making JEL classification: $\mathrm{O} 14$

Paper type: Research article

Received: Dec 01, 2017

Accepted: Feb 08, 2018

Citation: Erdogan, G., Refsdal, A., Nygård, B., Petter Rosland, O., Kvam Randeberg, B. (2018), "Risk-Based Decision Support Model for Offshore Installations", Business Systems Research, Vol. 9, No. 2, pp. 55-68.

DOI: 10.2478/bsrj-2018-0019

Acknowledgments: This work has been conducted within the AGRA project (236657) funded by the Research Council of Norway. The authors would like to thank Ketil Stølen for valuable feedback. 


\section{Introduction}

During the autumn of 2016, we developed a computerized model to support decisions based on operational safety risk offshore. The model automatically provides a decision advice based on 28 input parameters, and was developed using DEXi (Bohanec, 2017), which is a tool for multi-criteria decision modeling. The choice of DEXi was made based on early experience two of the authors had from using DEXi in the domain of cyber-risk (Refsdal et al., 2017; Erdogan et al., 2018).

The contribution of this paper is a report on our experiences, the efforts spent on the model development, as well as a presentation of the model and an initial evaluation. The aim is to help others who face related challenges to consider whether a similar approach may be suitable for them. We start by explaining the challenge and our success criteria.

During major maintenance projects on offshore installations, flotels are often used to accommodate the personnel. A flotel ("floating hotel") is a vessel providing sleeping quarters and other facilities. As illustrated in Figure 1, a gangway connects the flotel to the installation. The flotel needs to keep its position in a very limited area close to the installation. This can be done by means of Dynamic Positioning (DP), thruster assisted mooring or mooring systems. DP implies employing a computercontrolled system that allows the flotel to automatically keep its position by using its own thrusters. However, keeping the position is challenging due to the weather, waves, and other conditions offshore.

Figure 1

Gangway connection from flotel to installation

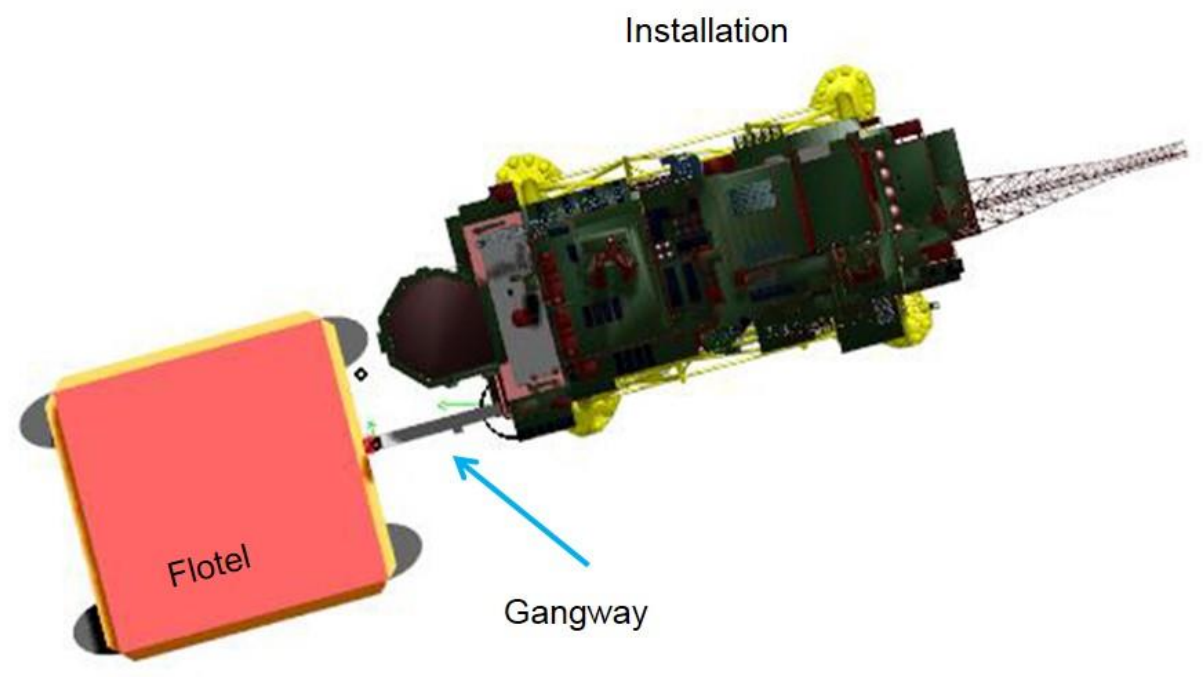

Source: Authors' work

If conditions are unfavorable, the responsible offshore operatives need to decide whether to lift (disconnect) the gangway from the installation. If this is not done, there is a risk that an uncontrolled autolift (disconnection) occurs, causing harm to personnel and equipment. The decision is difficult because many different factors affect the risk. Moreover, lifting the gangway has high economic cost, as workers will be prevented from performing their tasks on the installation. Currently, the offshore operatives make use of paper-based Location Specific Operational Guidelines (LSOG), along with a number of other sources of information, e.g. the prevailing weather conditions and the weather forecast, to guide the decision. 
To provide alternative decision support, ease the information handling and reduce dependency on the experience, competence and mental state of the individuals on duty at any given time, we envision a solution where advice is automatically generated based on a wider range of input parameters compared to the LSOG. This solution is illustrated in Figure 2.

Figure 2

Vision for overall decision support solution

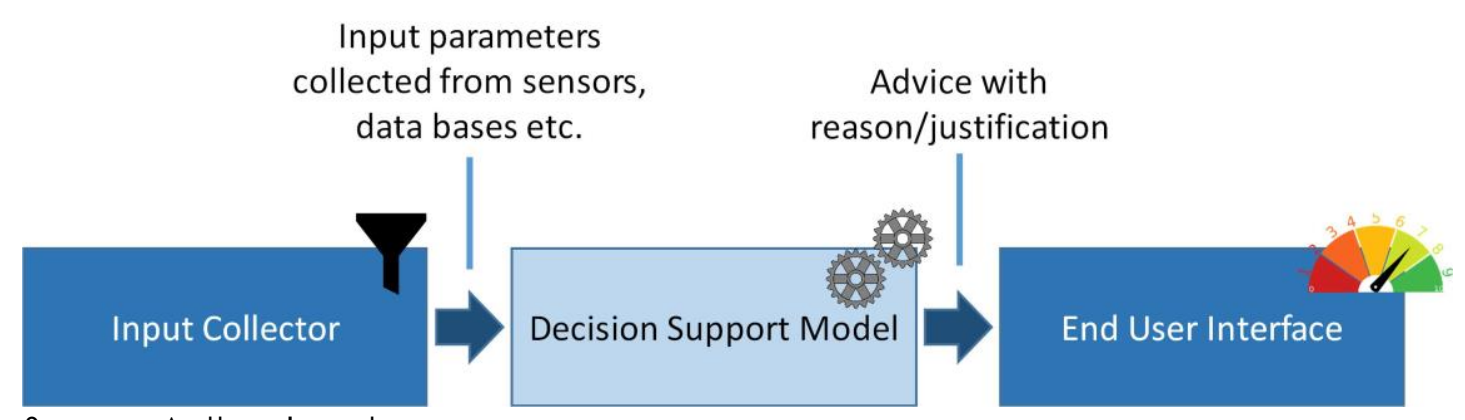

Source: Authors' work

The Input Collector collects all the data for the input parameters, such as weather forecasts and sensor readings. The Decision Support Model aggregates these data to compute an advice. This advice is presented in the End User Interface, which should be tailored to the human offshore operatives making the decision.

The work presented in this paper concerns the Decision Support Model. We identified the following success criteria for the model:

C1: The decision support model should provide advice that correspond with expert expectations.

C2: The decision support model should capture all aspects that are important for the assessment.

C3: The decision support model should be comprehensible for domain experts.

C4: The expected benefit should justify the effort required to develop the decision support model.

The rest of this paper is structured as follows. First, in Section 2 we introduce the DEXi tool, before explaining the method used for the development in Section 3. Section 4 presents the decision support model, while Section 5 presents expert feedback on the model. In Section 6 we discuss and evaluate the model with respect to criteria C1-C4. In Section 7, we present related work, before concluding in Section 8.

\section{The DEXi Tool}

DEXi (Bohanec, 2017) is a computer program for the development of multi-criteria decision models and the evaluation of options. Multi-criteria (also called multiattribute) models are a class of models used for decision analysis that evaluate options according to several, possibly conflicting, goals or objectives. In this section, we introduce DEXi, focusing on the parts needed to understand the rest of this paper. The reader is referred to the DEXi User Manual for further details on the tool (Bohanec, 2015).

Multi-attribute models decompose decision problems into a tree (or an acyclic graph) structure. The top attribute in such models represents the overall problem, while the attributes below the top attribute represent sub-problems, which are 
smaller and less complex than the overall problem. Each attribute is assigned a value. The set of values for an attribute is referred to as the scale of the attribute. DEXi supports definition of discrete ordinal scales, where each step typically consists of a textual description. An example of an ordinal scale is \{Unacceptable; Acceptable; Good; Excellent\}.

The attributes in the model are either basic or aggregate attributes. Basic attributes represent the inputs to the multi-attribute model. They have no child attributes. The value of a basic attribute is determined solely by the input to (or selected value for) the attribute.

Aggregate attributes have child attributes (which may be basic or aggregate). The value of an aggregate attribute is a function of the values of its child attributes. This function is called the utility function of the attribute. The utility function of each aggregate attribute is defined by stating, for each possible combination of its child attribute values, what is the corresponding value of the aggregate attribute.

In summary, developing a DEXi model implies the following:

- Identify the attributes and structure.

- Define the scale for each attribute.

- Define the utility function for each aggregate attribute.

For any given set of values for the basic attributes, the value assigned to the top attribute represents the overall aggregated evaluation.

\section{Method for Model Development}

As illustrated in Figure 3, we developed the model in four steps. In the first step, we established the context by identifying the purpose and scope, as well as deciding which tool to use. In the second step, we developed the decision support model by carrying out points i-iii described in Section 2. This was primarily done during video meetings where the analysis leader shared his screen and edited the DEXi model based on input and comments from the domain experts, while the analysis secretary took notes about the reasoning and discussions. Some modifications and corrections where also done offline, through email interaction.

Figure 3

Overview of method

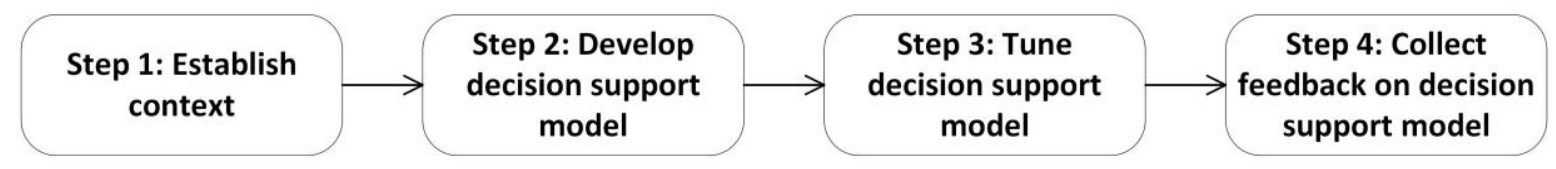

Source: Authors' work

In the third step, we tuned the decision support model by first defining a set of six scenarios based on the following criteria: 1) All scenarios should be realistic, i.e. represent conditions that might actually occur. 2) The set should include scenarios that cover all the possible decision alternatives defined in the LSOG. After describing the scenarios textually, each of the identified scenarios was translated to an assignment of a value to each basic attribute, referred to as an option in the DEXi tool. This allowed us to compare the advice produced by the model for each scenario with the guidelines provided by the LSOG. In cases of mismatch, we updated the DEXi model. In the fourth step, we collected feedback on the model, with focus on model structure and outcome for the six scenarios defined in the preceding step. 
As shown in Table 1, the above steps were carried out in 12 meetings held within a period of three months (from August 2016 to November 2016). All the authors took part in the model development. Of these, three are domain experts with technical experience within ship technology and marine systems in the petroleum industry, as well as software systems to support the petroleum industry with respect to risk-based decision-making. The remaining two (from SINTEF) served as analysis leader and secretary. The fourth step, i.e. feedback on the model, took place in meeting 12 (with preparations in meeting 11). The feedback was collected from three offshore operatives who represented the target group and who had not participated in developing the model or been involved in any other way before meeting 12. The feedback is explained further in Section 5. All meetings except meeting 12 were video meetings, while the 12th meeting was a combined physical and video meeting where one of the offshore operatives participated remotely from an offshore location. Although the steps are presented chronologically, they were sometimes revisited to make updates and adjustments or to capture new factors that were brought forward by the domain experts. Roughly speaking, the first step took place in meeting 1 and meeting 2, the second step took place from meeting 3 to meeting 7 , the third step took place from meeting 8 to meeting 10 , and the fourth step took place in meeting 11 and meeting 12.

Table 1

Overview of meetings

\begin{tabular}{|c|c|c|c|c|}
\hline M & Date & D & $\mathbf{S}$ & Activity \\
\hline 1 & 25.08 .16 & $1.5 \mathrm{~h}$ & 1 & Establish context \\
\hline 2 & 16.09 .16 & $2.5 \mathrm{~h}$ & 1 & $\begin{array}{l}\text { Finalize context establishment, present DEXi and progress } \\
\text { plan, and develop initial model structure }\end{array}$ \\
\hline 3 & 22.09 .16 & $2 \mathrm{~h}$ & 2 & Continue developing model structure \\
\hline 4 & 06.10 .16 & $2 \mathrm{~h}$ & 2 & $\begin{array}{l}\text { Complete model structure, define scales for attributes and } \\
\text { utility functions for aggregate attributes }\end{array}$ \\
\hline 5 & 13.10 .16 & $3 \mathrm{~h}$ & 2 & Continue defining attribute scales and utility functions \\
\hline 6 & 25.10 .16 & $3 \mathrm{~h}$ & 2 & Continue defining attribute scales and utility functions \\
\hline 7 & 27.10 .16 & $2 \mathrm{~h}$ & 2 & Complete defining attribute scales and utility functions \\
\hline 8 & 02.11 .16 & $2 \mathrm{~h}$ & 3 & Perform model tuning \\
\hline 9 & 11.11 .16 & $2.5 \mathrm{~h}$ & 3 & Perform model tuning \\
\hline 10 & 24.11 .16 & $2 \mathrm{~h}$ & 3 & Complete model tuning \\
\hline 11 & 28.11 .16 & $1 \mathrm{~h}$ & 4 & Prepare feedback collection \\
\hline 12 & 30.11 .16 & $6 \mathrm{~h}$ & 4 & Collect feedback from offshore operatives \\
\hline
\end{tabular}

Note: $M=$ Meeting, $D=$ Duration, $S=S t e p$ in method

Source: Authors' work

\section{The Decision Support Model}

In this section, we present the decision support model. We focus on the model structure, i.e. the attributes and the way in which these have been organized. The structure is shown in Figure 4, which have been extracted from the DEXi tool and presents the model as it was at the end of the development process, before meeting 12. As can be seen from the figure, the tool allows the user to provide a description in addition to the name for each attribute, which we made use of for most of the attributes. The remainder of this section explains the main characteristics of the model structure in further detail. 
Figure 4

The DEXi model structure

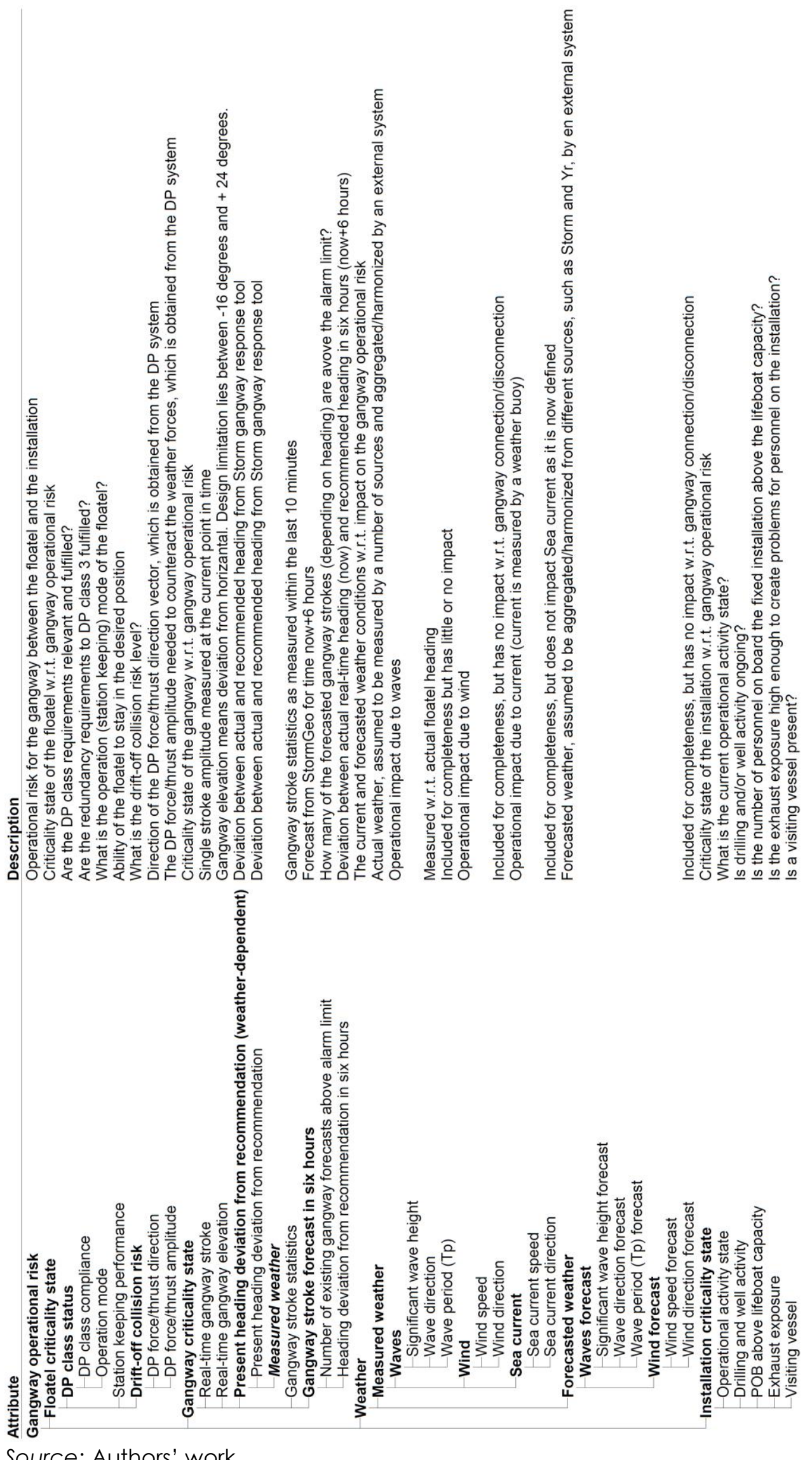


Gangway operational risk is the top attribute. The value assigned to this attribute represents the advice to the decision maker, since this advice depends directly on the risk level. Table 2 shows the scale for the attribute, and hence all the possible advice that can be produced by the model. The values are presented in order of decreasing risk, meaning that the value "Abandon operation" in the scale represents the least desirable state. Notice that to abandon operation in this context means to lift (disconnect) the gangway.

Table 2

Scale for the top attribute Gangway operational risk

\begin{tabular}{ll}
\hline Value & Description \\
\hline Abandon operation & $\begin{array}{l}\text { There are very strong reasons for disconnecting the gangway; an } \\
\text { autolift or other incident is likely. }\end{array}$ \\
$\begin{array}{ll}\text { Prepare to abandon } \\
\text { operation }\end{array}$ & $\begin{array}{l}\text { There are strong reasons for disconnecting the gangway. } \\
\text { Preparations for disconnection should be considered. }\end{array}$ \\
Advisory state & $\begin{array}{l}\text { If already disconnected, the gangway should remain so. If it is } \\
\text { currently connected, it may remain connected. }\end{array}$ \\
\hline Normal state & The gangway may safely be (or remain) connected. \\
\hline
\end{tabular}

Source: Authors' work

As shown in Figure 4, the top attribute has four child attributes, each representing one of the main categories of factors that affect the risk level. The first of the main categories is Flotel criticality state, which capture the factors related to the flotel. DP class compliance refers to the requirements to the Dynamic Positioning (DP) system, which is the control system used to maintain the desired position of the flotel using thrusters. The DP class compliance attribute captures the degree to which the requirements for redundancy of sensors, actuators and controllers in the DP system is fulfilled, since loss of redundancy imply a higher risk of not being able to control the flotel's position. The Operation mode attribute records whether the flotel is currently kept in position by using only the DP system, thruster assisted mooring (which combines the use of the DP system and mooring) or by using only mooring. Since the importance of fulfilling the DP system redundancy requirements depends on the degree to which the DP system is currently used to keep the flotel in position, the DP class status attribute aggregates DP class compliance and Operation mode. Station keeping performance captures the degree to which the flotel has been able to stay within the preferred position and heading (direction with respect to the installation) recently. The last attribute under Flotel criticality state is Drift-off collision risk, which represents the risk of the flotel drifting out of the desired position and colliding with the fixed installation. This risk depends on the direction and amplitude of the forces from the thrusters required to counteract the forces from waves, wind, etc., which is registered by the DP system and captured by the attributes DP force/thrust direction and DP force/thrust amplitude.

The second of the main categories is Gangway criticality state, which captures the factors related to the gangway between the flotel and the installation. The first two attributes in this category, i.e. Real-time gangway stroke and Real-time gangway elevation, capture the stroke and elevation of the gangway at the current point in time. Both are registered by sensors on the gangway, and should stay within fixed limits. Next, Present heading deviation from recommended heading (weather dependent) captures the degree to which the heading of the flotel is within the recommended limits, but compensating for the fact that the importance of staying within these limits depends on the weather. Notice that the Measured weather 
attribute under Present heading deviation from recommended heading (weather dependent) is a so-called linked attribute, which means that it occurs more than once in the structure. We come back to the Measured weather attribute below. Gangway stroke statistics captures the number of times the gangway stroke has been outside its fixed limit during the last 10-minute period. The final attribute under Gangway criticality state is Gangway stroke forecast in six hours, which depends on gangway stroke forecasts from a separate stroke prediction system and the expected deviation from the recommended heading in the next six hours, which is also obtained from an external system.

The third of the main categories is Weather, which is further decomposed into Measured weather and Forecasted weather. The former captures the current weather as measured by available sensors and services, while the latter captures the forecasted weather for the immediate future. Since weather data and forecasts can come from various sources, they do not necessarily give a consistent picture. We assume that harmonization of conflicting weather data is performed before assigning values to the relevant model attributes, as this is a challenge that is beyond the scope of the work presented here.

The fourth and final of the main categories is Installation criticality state, which captures the factors related to the installation connected to the flotel via the gangway. The Operational activity state attribute captures the operational activity on the installation, as the risk may depend on whether production or maintenance activities are currently ongoing. The same applies for Drilling and well activity. POB above lifeboat capacity captures whether there are currently more personnel on the installation (Personnel on Board) than there is room for in its life boats. This is relevant because the ability to escape via the gangway is extra important if the lifeboat capacity is exceeded. The Exhaust exposure attribute is included because exhaust may, in extreme cases, potentially cause problems for the personnel so that they need to leave the area. Finally, the Visiting vessel captures the presence of supply boats or other vessels, as this may have impact on collision risk and the available space to maneuver.

\section{Feedback on the Model}

In order to validate the model, we wanted to involve offshore operatives who belong to the target group and who had not taken part in the model development. Three such operatives were able to participate, of which two participated remotely. In addition, all those who had taken part in the model development were present.

The choice of validation approach was made based on practical considerations, in particular availability of key persons, time available, and the fact that not all participants would be physically present. The validation was carried out in a single meeting. Moreover, since some of the participants had no knowledge about the project context, DEXi, or the overall envisaged solution, we had to spend the initial part of the meeting introducing these concepts.

Given the practical restrictions, we decided to take a lightweight validation approach. The evaluation focused on the model structure and the outcomes for the six validation scenarios. The participants were asked to provide their opinions on both the structure and outcomes after a presentation from the leader of the meeting. Responses were primarily requested from the offshore operatives, but all participants took part in the discussions. The following questions were asked to initiate and guide the discussion: 
- Model structure:

- Are there any important parameters that are omitted?

- Are there any parameters that should be removed?

- Are the parameters properly organized?

- Outcome for selected scenarios:

- Does the model offer good recommendations for each scenario?

For the second part of the evaluation (outcome for selected scenarios), one of the domain experts who took part in the development of the model had defined, as part of the preparations for the meeting, six scenarios to represent in the model. These had been selected to cover cases that address several aspects while being realistic and interesting, and had been approved by another domain expert. The same scenarios had been used in the final phase of the model development to tune the model, in particular the scales and utility functions.

All participants agreed on the overall model structure. At the detailed level, we received three suggestions for additional attributes to be considered as descendants of one of the four existing attributes directly under Gangway operational risk. In addition, there was one suggestion to remove the Exhaust exposure attribute, as it was judged to have little impact on the decision on whether to lift the gangway. Finally, there was one attribute (DP class compliance) for which a refinement of the scale was proposed in order allow a more fine-grained distinction between states than our original scales offered.

With respect to the outcomes for selected scenarios, the offshore operatives were asked whether they agreed with the advice produced by the model for the scenarios. They unanimously agreed for five of the six scenarios. For the sixth scenario, where the advice provided by the model was Abandon operation, two expressed doubt or disagreement, even though the advice was consistent with the LSOG. The offshore operatives emphasized that the LSOG represents guidelines, rather than a set answer. One of the operatives said that he had experienced a similar scenario earlier. He then prepared to lift the gangway, but called a meteorologist to check the expected weather before making the decision. Since the weather was decreasing, they did not lift the gangway.

\section{Discussion and Evaluation}

Based on our experience, we now discuss and evaluate the fulfillment of criteria $\mathrm{Cl}$ C4 defined in Section 1.

C1 (The decision support model should provide advice that correspond with expert expectations): In our context, expert expectations are represented by the opinions of the offshore operatives taking part in the evaluation in the final meeting, as well as the LSOG, which is based on expert knowledge. As explained in Section 3, we made sure that the advice produced by the model were consistent with the LSOG for the identified scenarios. DEXi proved to have the expressive power to achieve this without any problems. For the one scenario where the offshore operatives did not agree with the model, the disagreement was due to a discrepancy between the guidelines in the LSOG and the opinions of the offshore operatives. Hence, the contended scenario is actually an issue of resolving discrepancy between different experts. We consider our results promising, although a thorough evaluation of criterion $\mathbf{c} 1$ requires a more extensive validation, preferably using more scenarios based on historical data, as well as involving more domain experts.

C2 (The decision support model should capture all aspects that are important for the assessment): The feedback on the model showed that the offshore operatives 
agreed with the overall structure and attributes. Incorporating their proposed modifications would not be a problem. Hence, we are confident that all the factors that the domain experts identified can be captured in the model. The aspects covered by the LSOG, which represent the current solution, is a proper subset of the aspects covered by the model.

However, one aspect not captured by the model is uncertainty. For example, input parameters, such as the weather forecast, are more or less uncertain. Even though the weather services provide an assessment of the uncertainty, this is ignored by the model. We considered including and aggregating uncertainty in the model, so that the advice offered as output could be accompanied by an aggregated assessment of the uncertainty. However, we saw no way to achieve this without significantly complicating the model, and the LSOG does not address the uncertainty of its guidelines. We therefore decided not to include uncertainty in the model.

While discussing $\mathbf{C 2}$, it is also interesting to touch on the issue of scalability. The most important aspect in this respect seems to be the size of the utility function for each attribute, i.e. number of possible combinations of values for its child attributes. This is determined by the number of child attributes and the granularity of their scales. The DEXi manual states that defining a utility function is quite hard for a size of 100 (Bohanec, 2015). In our model, the largest utility function, which belongs to the top attribute Gangway operational risk, has size 144. For this attribute, it was not acceptable to reduce the number of the child attributes, as the structure illustrated by Figure 4 was considered most appropriate. Reducing the number of steps in the scales was also not acceptable. We found the size 144 to be manageable, due to functionality that DEXi offers for checking consistency of a utility function and automatically suggesting possible values for missing entries based on already inserted entries. Still, we believe that utility functions larger than ca. 150 would be highly impractical.

C3 (The decision support model should be comprehensible for domain experts): This criterion implies that the domain experts should be able to understand the algorithm by inspecting the model. This increases trust in the outputs from the model, and means that the model can also facilitate knowledge sharing and learning. None of the domain experts who participated in the model development had any knowledge about DEXi before the process. Even so, after a brief introduction, they quickly grasped the DEXi concepts and were able to contribute to the model development. Throughout the process, the comments, suggestions and discussions demonstrated that all participants were able to understand the details of the evolving model. Thus, we avoided the misunderstandings and problems typically encountered when an executable algorithm is implemented in a language not understood by the domain experts. Basically, the DEXi model served as a combined specification and implementation of the assessment algorithm that was fully transparent for all participants.

C4 (The expected benefit should justify the effort required to develop the decision support model): Our estimate indicates that the model development amounts to ca. 150 person hours in total. This includes ca. 100 hours spent on meetings 2 to 11 . The estimate does not include meetings 1 and 12, as no model development or updates were done in these meetings. Ca. 50 hours was spent on work between meetings. Of the latter, ca. 16 hours was spent by the domain experts on checking the model and defining scenarios, while the remaining 34 hours was spent by the analysis leader and secretary on taking notes and correcting the model. We are not aware of other works reporting on the effort required to develop this type of model. However, for the 
model-based risk analysis method CORAS (Lund et al., 2011), the authors state that the expected effort required to carry out a CORAS analysis is typically from 150 to 300 hours. This gives at least an indication that the amount spent on developing our decision support model is reasonable.

Of course, a thorough evaluation of criterion C4 would require that we quantify the benefit, as well as the cost. This is very hard, and we have not attempted to do so. Still, we believe that the benefit justifies the effort. First, the model produces consistent advice which may be a valuable supplement to a largely experience based decision-making process. There is also a potential for reuse of (parts of) the model to support related decisions. Second, the process of developing the model collectively in a group creates learning and raises the awareness of those taking part. Third, the resulting model codifies and documents knowledge from all those taking part in the development, thus serving as a vehicle for knowledge transfer throughout the organization. While the first point was a central part of our motivation for initiating the work, and known in advance, the added benefit of the last two points became clear to us during the process.

Given the practical limitations and approach for obtaining feedback on the model, there are some obvious threats to validity. Since only three offshore operatives who had not taken part in the development of the model took part in the validation, the feedback we received was very dependent of these particular individuals. On the positive side, all three were in the core target group for the envisaged solution.

Only six scenarios were considered for evaluation of the advice provided by the model. Since the model includes 28 basic attributes, each with two or more possible values, the six scenarios cover a small proportion of the theoretically possible combinations of values for basic attributes. However, the six scenarios had been carefully selected by one domain expert (and approved by another) to cover cases that address a wide range of aspects while being realistic and interesting. For each scenario, the model outcomes were shown to the three offshore operatives before they were asked whether they agreed. Such an approach implies that there is a chance that the respondents give a confirmative answer without thinking it through to arrive at their own conclusion. However, the expression of disagreement for one of the scenarios, for which clear arguments were provided, indicates that the participants did indeed take the effort to make up their own opinions.

\section{Related Work}

The field of multi-criteria decision making consists of many different approaches. Velasquez et al. (2013) present a comprehensive literature review addressing multicriteria decision making methods including Multi-Attribute Utility Theory (MAUT) (Fishburn, 1967; Fishburn et al., 1974; Keeney 1977), Simple Multi-Attribute Rating Technique (SMART) (Chen et al., 2010), and Analytic Hierarchy Process (AHP) (Saaty, 1980; Saaty, 2008). For each approach, they provide a description and present advantages, disadvantages, and area of application.

Compared to DEXi, the advantage of MAUT is the inclusion of uncertainty, while the disadvantage is that MAUT requires a very large amount of input at every step of the procedure to accurately capture the decision maker's preferences (Velasquez et al., 2013). The simple Multi-Attribute Rating Technique (SMART) is a simpler approach to MAUT and one of the main advantages of SMART is the flexibility to use any type of weight assignment techniques (i.e., relative, absolute, etc.). However, similar to MAUT, the procedure in SMART to determine weight coefficients is not convenient because of its complicated framework (Konidari et al., 2007). Like DEXi, 
the AHP is also easy to use and scalable in terms of adjusting the hierarchy structure to fit many sized problems. The disadvantage of AHP is related to interdependence between criteria and alternatives, which in turn may cause inconsistencies between judgement and ranking criteria (Konidari et al., 2007; Velasquez et al., 2013). This is possible to avoid in DEXi by making use of scale orders.

DEXi has been applied in many different domains. Some of these are "health care, finance, construction, cropping systems, waste treatment systems, medicine, tourism, banking, manufacturing of electric motors, and energy" (Bohanec et al., 2013; Bohanec, 2017). To the best of our knowledge, neither DEXi nor the abovementioned approaches (the latter is according to Velasquez et al. (2013)) have been used to assess safety risks within offshore as reported in this paper.

However, DEXi has been applied to assess safety risks within highway traffic (Omercevic et al., 2008) and ski resorts (Bohanec et al., 2015). These approaches use DEXi models as the underlying algorithm to compute an advice based on relevant input data and are thus similar to our approach. In particular, the approach provided by Omercevic et al. (2008) use DEXi models in a framework where input data is collected via sensors in the highway. This is in line with our envisioned automated solution illustrated in Figure 2. The details of the End User Interface and the Input Collector are beyond the scope of this paper and therefore not explained further. However, we are confident that our envisioned solution is feasible as we have in fact taken part in implementing a similar approach in a framework for realtime cyber-risk assessment (Refsdal et al., 2017) developed by the WISER-project (WISER, 2017). The focus of the WISER framework, however, is on cybersecurity risk and not safety risk. Moreover, the risk assessment in the WISER framework produces risk levels which indicate the severity of security risks, while in our approach the decision support model produces advice on whether to disconnect/connect the gangway from/to the installation. In addition to risk levels, the WISER framework also suggests risk treatments for the assessed risks, which are advice decision makers can use to select appropriate risk-mitigation strategies.

Unlike most of the existing publications on DEXi, we have focused on the overall approach, in addition to presenting details of the model. In particular, we address the efforts spent to develop the model, the involvement of domain experts, and the comprehensibility of the model, as well as the quality of the final result. These aspects are important for others who consider a similar approach.

\section{Conclusion}

In this paper, we shared our experiences from using DEXi to develop support for riskbased decisions for offshore flotels and presented the resulting model. The results indicate that the decision support model provides advice that correspond with expert expectations, captures all aspects that are important for the assessment, is comprehensible for domain experts, and that the expected benefit justifies the effort required to develop the model.

Threats to validity are mainly related to the practical limitations and approach for obtaining feedback on the model. The feedback was dependent of three offshore operatives, and only six scenarios were considered for evaluation of the advice provided by the model. However, on the positive side, the offshore operatives (who had not taken part in the development of the model) were all in the core target group for the envisaged solution, and the six scenarios had been carefully selected and approved by domain experts to cover cases that address a wide range of aspects while being realistic and interesting. 
Our motivation was to make others who face related challenges aware of the possibilities, and help them to consider whether a similar approach is suitable for their needs. We have focused on the issues that we think are of general relevance. Based on our experience and overall evaluation, we consider our results quite promising, and believe that the approach can be fruitful for a wider range of risk-based decisions. In future projects, we hope to explore these possibilities further.

\section{References}

1. Bohanec, M. (2015), "DEXi: Program for Multi-Attribute Decision Making. User's Manual version 5.00 IJS DP-11897", available http://kt.ijs.si/MarkoBohanec/DEXi/html/DEXiDoc.htm (14 November 2017).

2. Bohanec, M. (2017), "DEXi: A Program for Multi-Attribute Decision Making", available at: http://kt.ijs.si/MarkoBohanec/dexi.html (14 November 2017).

3. Bohanec, M., Delibasic, B. (2015), "Data-Mining and Expert Models for Predicting Injury Risk in Ski Resorts", in Delibasic, B. et al. (Eds.), Decision Support Systems V - Big Data Analytics for Decision Making, Springer, Cham, pp. 46-60.

4. Bohanec, M., Znidarsic, M., Rajkovic, V., Bratko, I., Zupan, B. (2013), "DEX Methodology: Three Decades of Qualitative Multi-Attribute Modeling", Informatica, Vol. 37, No. 1, pp. 4954.

5. Chen, Y., Okudan, G. E., Riley, D. R. (2010), "Decision support for construction method selection in concrete buildings: Prefabrication adoption and optimization", Automation in Construction, Vol. 19, No. 6, pp. 665-675.

6. Erdogan, G., Refsdal, A. (2018), "A Method for Developing Qualitative Security Risk Assessment Algorithms", in Cuppens, N. et al. (Eds.), Risks and Security of Internet and Systems, Springer, Cham, pp. 244-259.

7. Fishburn, P. C. (1967), "Conjoint measurement in utility theory with incomplete product sets", Journal of Mathematical Psychology, Vol. 4, No. 1, pp. 104-119.

8. Fishburn, P. C., Keeney, R. L. (1974), "Seven independence concepts and continuous multiattribute utility functions", Journal of Mathematical Psychology, Vol. 11, No. 3, pp. 294-327.

9. Keeney, R. L. (1977), "The art of assessing multiattribute utility functions", Organizational Behavior and Human Performance, Vol. 19, No. 2, pp. 267-310.

10. Konidari, P., Mavrakis, D. (2007), "A multi-criteria evaluation method for climate change mitigation policy instruments", Energy Policy, Vol. 35, No. 12, pp. 6235-6257.

11. Lund, M. S., Solhaug, B., Stølen, K. (2011), "Model-Driven Risk Analysis: The CORAS Approach", Springer, Berlin, Heidelberg.

12. Omercevic, D., Zupancic, M., Bohanec, M., Kastelic, T. (2008), "Intelligent response to highway traffic situations and road incidents", in Transport Research Arena Europe 2008, Ljubljana, pp. 21-24.

13. Refsdal, A., Erdogan, G. (2017), "D3.4 Cyber risk modelling language and guidelines", available at: https://www.cyberwiser.eu/ (14 November 2017).

14. Saaty, T. L. (1980), "The analytic hierarchy process: planning, priority setting, resources allocation", McGraw-Hill, New York.

15. Saaty, T. L. (2008), "Decision making with the analytic hierarchy process", International Journal of Services Sciences, Vol. 1, No. 1, pp. 83-98.

16. Velasquez, M., Hester, P. T. (2013), "An analysis of multi-criteria decision making methods", International Journal of Operations Research, Vol. 10, No. 2, pp. 56-66.

17. WISER (2017), "Wide-Impact cyber SEcurity Risk framework", available at: https://www.cyberwiser.eu/ (14 November 2017). 


\section{About the authors}

Gencer Erdogan, PhD, Research Scientist at SINTEF Digital. He received his PhD in computer science from the University of Oslo in 2016 with the dissertation thesis "CORAL: A Model-Based Approach to Risk-Driven Security Testing". His research interests include information and system security, modelling, model-based security and cyber-risk analysis, and model-based risk-driven security testing. Gencer Erdogan has broad experience from national and international research projects within the field of model-based specification and risk assessment, and risk-based security testing, as well as industrial experience as a software developer and security tester. The author can be contacted at gencer.erdogan@sintef.no.

Atle Refsdal, PhD, Senior Research Scientist at SINTEF Digital. He received his PhD in computer science from the University of Oslo in 2008 with the dissertation thesis "Specifying Computer Systems with Probabilistic Sequence Diagrams". He also holds a Master degree in Language, Logic and Information, as well as being a trained industrial electronics engineer. His research interests include model-based security and cyber-risk analysis, as well as formal specification and analysis. Atle Refsdal has broad experience from national and international research project within the fields of model-based specification and analysis, as well as several years of industrial experience as an engineer and software developer. The author can be contacted at atle.refsdal@sintef.no.

Bjørn Nygård, MSc, Advisor Platform Technology at Statoil. He received his MSc in Marin Technology from The Norwegian Institute of Technology (NTH, now NTNU) in December 1990. He has more than 25 years of experience within software development, project engineering and project management primarily for the maritime and offshore sectors. Bjørn Nygård is interested in, and has hands-on experience with, offshore and onshore audits, incident investigations, marine operations, writing technical specifications and user manuals, technology qualifications and risk assessments. The author can be contacted at bjnyg@statoil.com.

Bernt Kvam Randeberg, MSC, CEO at OTG Solutions AS. He received his MSc in Material / Offshore technology from University of Stavanger in 1993. He also holds a bachelor degree in Mechanical Engineering. He has 23 years of engineering experience within project management, software development as product owner, product design, R\&D, manufacturing and risk management for international oil \& gas companies and in the medical device industry. Bernt Randeberg has hands-on experience with offshore/onshore audits, investigations, GAP studies, barrier management, writing technical SW/hardware specifications, product verification, validation processes and has facilitated a large number of qualitative and quantitative risk analysis (HAZID / HAZOP / FMEA / ROS) across industries. The author can be contacted at bernt.kvam.randeberg@otg.no.

Ole Petter Rosland, MSc, Electronics Engineering. He is currently working for Norwegian energy company Statoil with flotel-operations. He received his MSc from the Norwegian Technical University of Trondheim (NTH) in 1981. He has worked for almost 20 years with the design of medical devices and medical training devices, both software and hardware. He started working in the Norwegian oil industry in 2007, first with $H S E Q$, and later with offshore operations. He has a special interest in risk management and the relation between probability based risk (historic, statistical risk) and uncertainty (future risk). The author can be contacted at olpr@statoil.com. 